\title{
A KNOWLEDGE BASED TWO-STAGE CASCADE MODEL FOR TEST-QUESTION/ANSWER RETRIEVAL
}

\author{
Yilu Wei, Daifeng Li and Andrew David Madden \\ School of Information Management, Sun Yat-sen University, GuangZhou, China
}

\begin{abstract}
Test-question/answer retrieval task has raised higher requirements in terms of accuracy, coverage and semantic understanding. We design a cascade model with two-stage training processes: The first stage uses 41,532 user test-question click records and 207,660 unclick records, which are collected from a designed test-question-answer experimental platform, to generate 200,000 pairwise training dataset to train a deep learning model, which could improve generalization ability. The second stage combines the output of the first stage with structural knowledge as new features to train a logistic regression for selecting the results from the candidates with higher accuracy, the training dataset is generated by manually annotating 20,000 test-question samples. The structural knowledge is also manually extracted from the samples for generating a small knowledge graph, and on this condition, we design knowledge features. Experimental results show that the proposed model outperforms the state-of-the-art algorithms, among which the cascading model contributes $3 \%$ improvement and the knowledge features contribute $1 \%$ improvement.
\end{abstract}

\section{KEYWORDS}

Deep Learning, Test-Question/Answer Retrieval, Knowledge Feature, Cascade Model

\section{INTRODUCTION}

In recent years, vertical domain search services have become more and more popular in China. One of the most popular domains is education. China has 200 million primary, secondary and college students who create strong demand for educational resources. Various search applications have been developed to address the students' needs for knowledge and answers. For example, Zuoyebang (https://www.zybang.com/) allows students of all disciplines to search for test-questions. Students can input their questions in the form of text, voice or images, and the system provides answers. Unlike traditional information retrieval tasks or relevance models, test-question searching has unique requirements, which are described below:

- Strong generalizability: Test-questions are often expressed in a diverse and complex manner. Some results have the same meaning as the target query, but are expressed in a different manner, so traditional term-matching based relevance models (Salton et al. 1975) do not work.

- Semantic understanding for high accuracy: A relevance model without semantic understanding may lead to serious misinterpretations (Gómez-Adorno et al. 2013). For example, "Length and width are known. How should area be calculated" versus "Length and width are known. How should perimeter be calculated" are very different, but it is hard to distinguish them without semantic understanding.

- Interpretable model: Deep Learning is hard to interpret, thus increasing the incidence of errors and bad cases. For test-question/answer tasks, errors are usually unacceptable because minor differences may lead to irrelevant answers. Proper understanding arising from correct interpretations is particularly important for test-question retrieval.

Much research has focused on improving the generalizability of existing relevance models, and improving their capacity for semantic understanding (Xiao et al. 2018, P. S. Huang et al. 2013). However, researchers have seldom conducted specific domain optimization using procedures such as combining users' behavior records with domain structural knowledge to design an interpretable model. Not only is it hard to design a complete model for accurate semantic understanding, it is also hard to generalize it to handle lots of complex semantic representations in reality. To achieve this, we designed a knowledge-based two-stage cascading model, the main innovations of which are listed as below: 
- The framework was designed to take into account both generalizability and accuracy. In the first stage, generalizability was improved by using a large-scale users' online behavior log. In the second stage, structural knowledge was used to obtain results that are more accurate.

- The first stage was achieved by use of a deep learning framework with pairwise strategy (Timur et al. 2019) which detected users' preference patterns from a training dataset based on 200,000 pairwise data. A multi-layer deep neutral network was designed on the framework. We selected word embedding (Danchen et al. 2018) to vectorize the input so that we could obtain the maximum fuzzy matching segment of title towards query.

- We designed knowledge features and used pointwise strategy to improve the capability of semantic understanding and to acquire results that are more accurate.

\section{THE FRAMEWORK OF THE PROPOSED MODEL.}

We designed a two-stage cascading model to handle the test-question/answer retrieval task. The framework is shown in Figure 1:

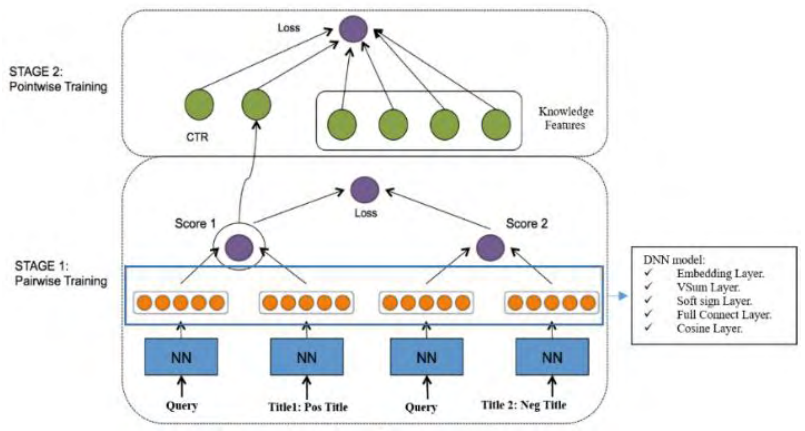

Figure 1. Framework of the two-stage cascading model

\subsection{Stage 1: Deep Neural Network Model with Pairwise Learning Strategy}

According to previous studies (Sunil et al. 2017, Jun et al. 2015), users' preferences and clicking behaviors show a strong semantic correlation. In this case, the dataset contains 200,000 pairwise training data, and we adopted a pairwise strategy. When different users entered the same query, we selected two candidate results, labelled Title 1 and Title 2. If the number of clicks on Title 1 was greater than that on Title 2 we classed Title 1 as a good title and Title 2 as a poor title, and a pairwise based partial order is generated. To eliminate the influence of ranking position COEC (Click Over Expected Clicks) was used (Zhang et al. 2007).

Score 1 is the relevance score between the query and Title 1, while Score 2 is the relevance score between the query and Title 2. A loss function is used to determine whether Score 1 is greater than Score 2. If Score 1 $>$ Score 2, then we consider that the DNN model has a correct understanding of relevance; If Score $1<=$ Score 2, the learned model has biases in understanding the relevance, and needs to be optimized through a back-propagation algorithm. The DNN model consists of 5 layers which are described in Figure 2. 


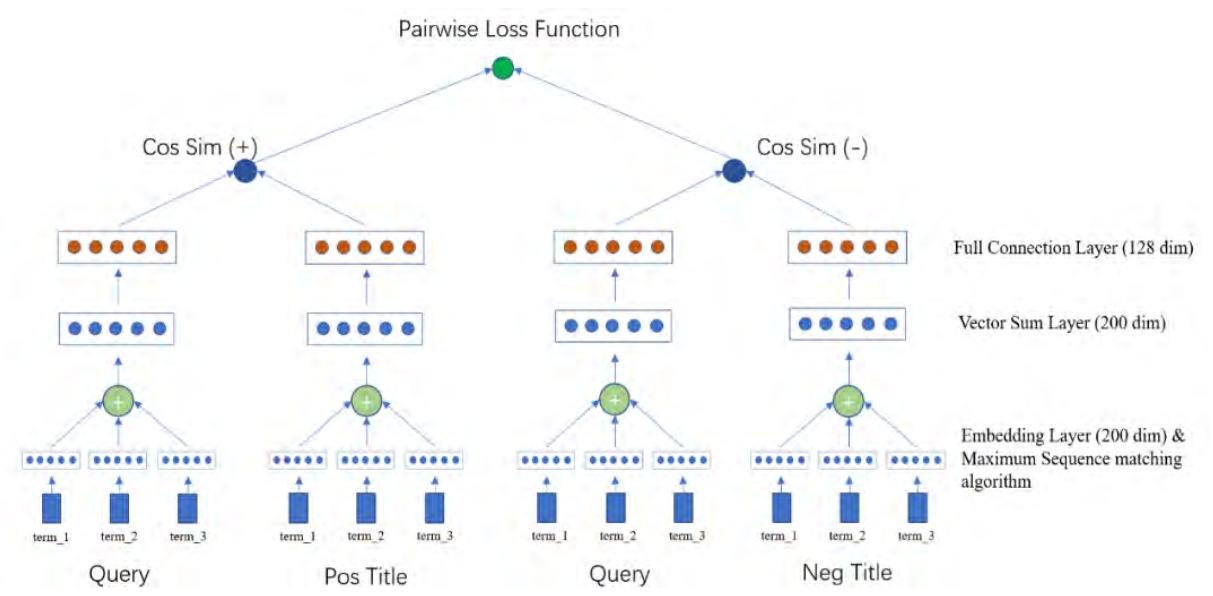

Figure 2. Framework of DNN with pairwise strategy

\subsubsection{Input Format and Embedding Layer}

For pairwise strategy, assume the input query $=\left\{\mathrm{q} \_t e r m_{1}, \mathrm{q} \_\right.$term $_{2}, \mathrm{q} \_$term $_{3}, \ldots \mathrm{q} \_$term $\left.\mathrm{q}\right\}$, the positive title is posTitle $=\left\{p_{-}\right.$term $1, p_{-}$term $2, p_{-}$term $3, \ldots p_{-}$term $\left.{ }_{p}\right\}$, the negative title is negTitle $=\left\{\mathrm{n}_{-}\right.$term $1, \mathrm{n}_{-}$term $2, \mathrm{n}_{-}$term 3 , $\ldots \mathrm{n} \_$term $\left.\mathrm{n}\right\}$. In the embedding layer, we use word 2 vec to vectorize each term of query, posTitle and negTitle, which can be seen in formulas (1), (2), and (3):

$$
\begin{aligned}
& q_{-} \text {term }_{i} \stackrel{\text { Embedding }}{\longrightarrow} V q_{i}=\left\{q_{i 1}, q_{i 2}, \ldots \ldots, q_{i k}\right\} \\
& p_{-} \text {term }_{i} \stackrel{\text { Embedding }}{\longrightarrow} V p_{i}=\left\{p_{i 1}, p_{i 2}, \ldots \ldots, p_{i k}\right\} \\
& n_{-} \text {term }_{i} \stackrel{\text { Embedding }}{\longrightarrow} V n_{i}=\left\{n_{i 1}, n_{i 2}, \ldots \ldots, n_{i k}\right\}
\end{aligned}
$$

where $i$ is the $i$ th term of query, posTitle or negTitle. $\mathrm{q}_{i j}, \mathrm{p}_{i j}, \mathrm{n}_{i j}$ are the value of $j$ th dimension. $k$ is the dimension of vector (which is set to 200 here). The configuration of the embedding layer can be seen below:

embedding_layer $=\{$ type: "embedding",

learn strategy: "word2vec",

parameter: "embedding param",

input: [query, posTitle, negTitle],

output: [query embedding, posTitle embedding, negTitle embedding]\}

\subsubsection{Vector Sum Layer}

In Vector Sum Layer, we use formulas (4), (5), and (6) to obtain vector representation of query and title, which are listed below:

$$
\begin{aligned}
& q_{-} v=\sum_{i=1}^{q} V q_{i}=\left\{\sum_{i=1}^{q} q_{i 1}, \sum_{i=1}^{q} q_{i 2}, \sum_{i=1}^{q} q_{i 3}, \ldots \ldots \sum_{i=1}^{q} q_{i k}\right\} \\
& p_{-} v=\sum_{i=1}^{p} V p_{i}=\left\{\sum_{i=1}^{p} p_{i 1}, \sum_{i=1}^{p} p_{i 2}, \sum_{i=1}^{p} p_{i 3}, \ldots \ldots \sum_{i=1}^{p} p_{i k}\right\} \\
& n_{-} v=\sum_{i=1}^{n} V n_{i}=\left\{\sum_{i=1}^{n} n_{i 1}, \sum_{i=1}^{n} n_{i 2}, \sum_{i=1}^{n} n_{i 3}, \ldots \ldots \sum_{i=1}^{n} n_{i k}\right\}
\end{aligned}
$$

where $\mathrm{q}_{-} \mathrm{v}$ represents the sum of query term vector, $\mathrm{p}_{-} \mathrm{v}$ represents the sum of posTitle term vector and $\mathrm{n}_{-} \mathrm{v}$ represents the sum of negTitle term vector. The configuration of Vector Sum Layer can be seen as below:

vector_sum_layer $=\{$ type: "vector sum", parameter: "vector_sum param",

input: [query embedding, posTitle embedding, negTitle embedding], output: [query emb sum, posTitle emb sum, negTitle emb sum]\} 
In addition, we designed a maximum sequence-matching algorithm to find the most related pattern of title towards query. The algorithm is displayed below:

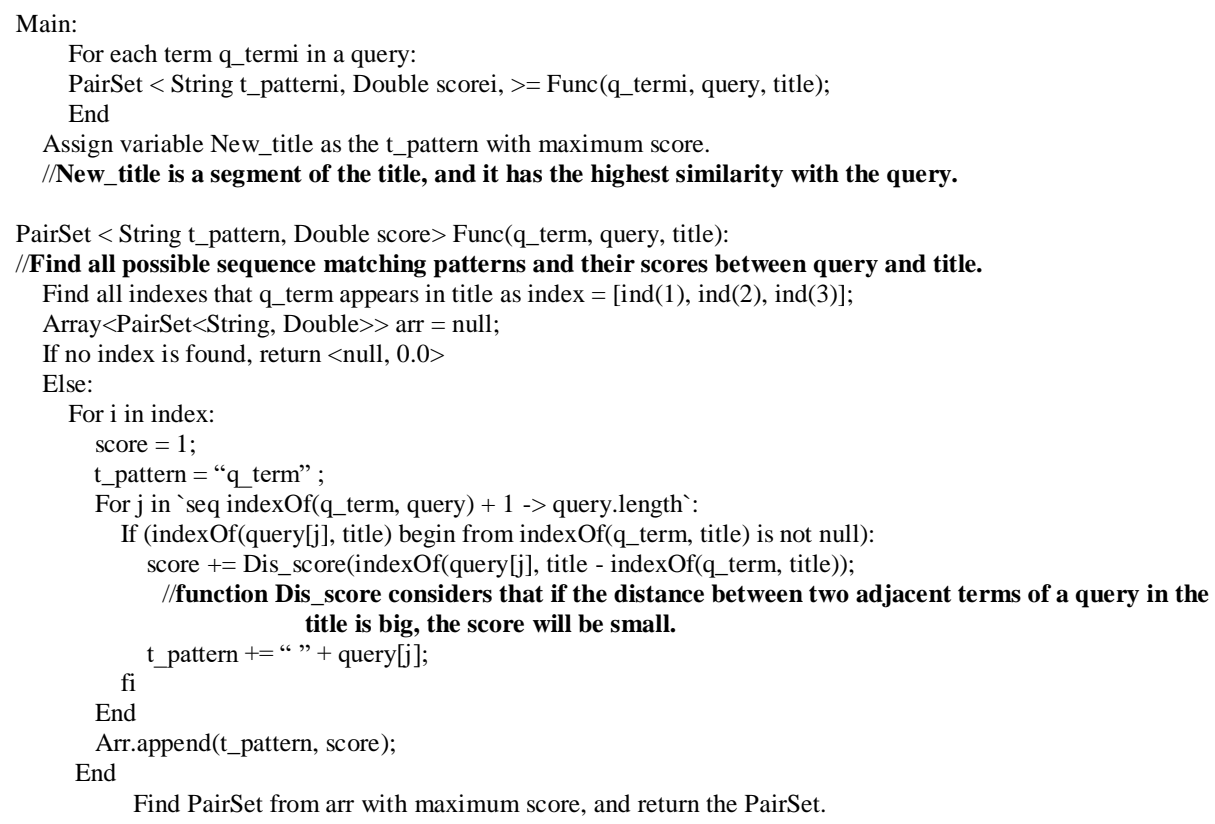

Algorithm 1. Maximum sequence-matching algorithm for finding the most suitable title segment for the target query

\subsubsection{Fully Connected Layer}

We assign a 128-dimension vector of neurons in a fully connected layer, and map the 200-dimension embedding vector into the 128-dimension vector (Hinton et al. 2006). In the fully connected layer, all queries share the same parameter distributions and all titles share the same parameter distributions. In the embedding layer, we use a pre-training strategy to obtain the vectored representation of each term. The configuration of fully connected layer can be seen below:

full_connect_layer = type: "full connect",

learning strategy: "full connection",

parameter: "full connect param",

input: [query emb sum, posTitle emb sum, negTitle emb sum],

output: [query fc, posTitle fc, negTitle fc]\}

\subsubsection{Cosine and Loss Function Layer}

In the cosine layer, we use a cosine function to calculate the cosine similarity (Metzler et al. 2005) between query and posTitle, and between query and negTitle. In the loss function layer, we select Hinge Loss function. The detail of the network design is shown below:

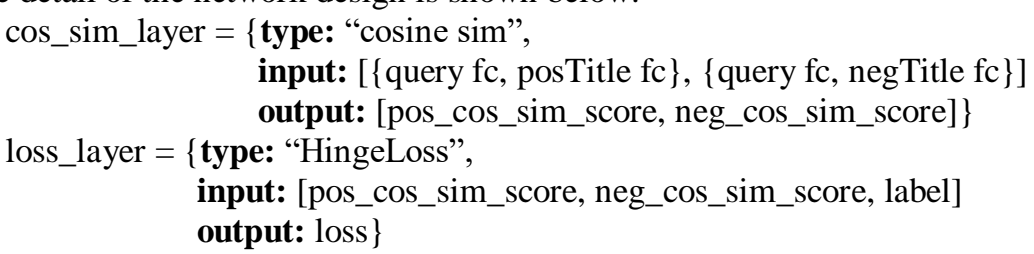

network $=\{$ embedding layer, vector sum layer, full connect layer, cos_sim_layer, loss_layer $\}$

\subsection{Stage 2: Knowledge Feature Design for Accurate Semantic Understanding}

Scores obtained from Stage 1 were used to improve generalizability. In addition, we added features to improve the model's capacity for semantic understanding. Added features included entity-attribute (which was extracted 
from the condition description of a question), and the target solution of the question. We manually extracted entity-attribute and entity-action from the samples to generate a small knowledge graph that covers 7 disciplines (Chinese, math, chemistry, physics, biology, history and geography). The knowledge graph contains 2,582 entities, 6,336 attributes and 1,213 actions. For math related questions, we defined three patterns to identify different target solutions. These are summarized below:

Pattern 1: “求” (request) + [entity][attribute] | [entity] | [attribute] + “?” | “”

Pattern 2: [entity][attribute] | [entity] | [attribute] + “怎么求” (how to find out) | “?” | “”

Pattern 3: [entity][attribute] | [entity] | [attribute] + “是” | “重” | “有” (is | has) | “” + “多少” (how much) + [unit] | “” + “?”| “”

We use the extracted entity-attribute-action to build the knowledge graph, then use the knowledge graph to generate knowledge features. The main principle is that we use the knowledge graph and a NLP parse method to extract entities, attributes, attribute values and actions for both query and title. We then analyze whether query and title describe the same entity with the same attribute or action, for the same attribute, and whether they have the same value. The comparison results can be seen as knowledge features, and gets assigned to a sequence of feature slots. Details of the knowledge feature generation can be seen below in Figure3.

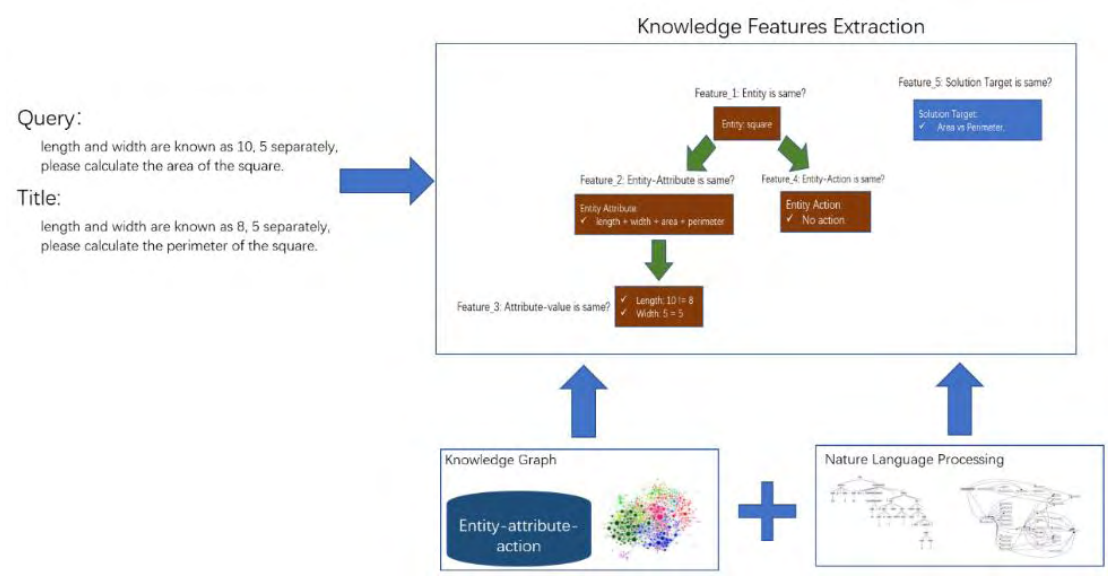

Figure 3. Examples of knowledge extraction features.

\section{DATA}

Table 1. Data Format of test-question/answer searching records

\begin{tabular}{|c|c|c|c|c|c|}
\hline Userid & Query & Title & Answer & Rank & Click \\
\hline 1 & $\begin{array}{l}\text { using } 30 \text { stamps with } \\
\text { a length of } 3 \mathrm{~cm} \text { and a } \\
\text { width of } 2.5 \mathrm{~cm}\end{array}$ & $\begin{array}{l}\text { Use } 30 \text { stamps that have } \\
\text { a length of } 3 \mathrm{~cm} \text { and a } \\
\text { width of } 2.5 \mathrm{~cm} \text { to form a } \\
\text { square. What is the } \\
\text { length of the square' } \mathrm{s} \\
\text { sides? }\end{array}$ & $\begin{array}{l}\text { Using } 30 \text { stamps with a length of } 3 \mathrm{~cm} \\
\text { and a width of } 2.5 \mathrm{~cm} \text { to form a square } \\
\text { without gaps, the area of every stamp is } \\
\text { calculated first, and then the total area of } \\
\text { the square. After that, the square root of } \\
\text { the area is just the length of sides. }\end{array}$ & 2 & 1 \\
\hline 2 & $\begin{array}{l}\text { Oxygen production } \\
\text { equation with } \\
\text { hydrogen peroxide }\end{array}$ & $\begin{array}{l}\text { How to write equation } \\
\text { for oxygen production } \\
\text { from hydrogen peroxide }\end{array}$ & $2 \mathrm{H}_{2} \mathrm{O}_{2}=\mathrm{MnO}_{2}=2 \mathrm{H}_{2} \mathrm{O}+\mathrm{O}_{2} \uparrow$ & 3 & 1 \\
\hline 3 & $\begin{array}{l}\text { When a car suddenly } \\
\text { starts, passengers } \\
\text { will move backward, } \\
\text { because they are } \\
\text { under the force of } \\
\text { inertia }\end{array}$ & $\begin{array}{l}\text { "When a car suddenly } \\
\text { starts, passengers will } \\
\text { move backward, because } \\
\text { they are under the force } \\
\text { of inertia". Why is this } \\
\text { sentence wrong? }\end{array}$ & $\begin{array}{l}\text { It should not be "under the force of } \\
\text { inertia", because inertia is an inherent } \\
\text { property of objects. The original } \\
\text { sentence should be changed to: when a } \\
\text { car suddenly starts, passengers will } \\
\text { move back because they have inertia. }\end{array}$ & 1 & 1 \\
\hline
\end{tabular}


The experimental dataset, which mainly comprises records relating to users' searching and clicking behavior in the test-question/answer domain, is collected from a vertical domain experimental platform. We crawled 325,415 questions from websites, which cover 7 disciplines, and then built the test-question-answer platform by using a term-matching cosine algorithm. Participants were invited to use the platform and provided clicking data, allowing us to collect 41,532 records relating to user searching and clicking, and 20,7660 unclicked records for pairwise comparison. The format of each record is described in Table 1.

The format used to label the 20,000 samples from the 41,532 records is shown in Table 2. For each sample, the following were manually extracted: entity-attribute; entity-action; target from query; result. Queries and titles were then compared from two perspectives: target matching (same/not same/unknown) and relevance (Rel). Relevance is on a scale from 0 to 2, where 2 is the most relevant. We also used patterns defined above to formalize the math-related solution target.

Table 2. Manual labeling format of the selected records

\begin{tabular}{|c|c|c|c|c|c|c|c|}
\hline id & Query & Target & Title & $\begin{array}{l}\text { Entity } \\
\text {-Attribute } \\
\text {-Action }\end{array}$ & Target & $\begin{array}{l}\text { Target matching } \\
\text { (same/not } \\
\text { same/unknown) }\end{array}$ & Rel \\
\hline 1 & $\begin{array}{l}\text { using } 30 \text { stamps } \\
\text { with a length of } \\
3 \mathrm{~cm} \text { and a width of } \\
2.5 \mathrm{~cm}\end{array}$ & NULL & $\begin{array}{l}\text { Use } 30 \text { stamps that have } \\
\text { a length of } 3 \mathrm{~cm} \text { and a } \\
\text { width of } 2.5 \mathrm{~cm} \text { to form a } \\
\text { square. What is the } \\
\text { length of the square' } \mathrm{s} \\
\text { sides? }\end{array}$ & $\begin{array}{l}\text { Entity: square } \\
\text { Attribute: } \\
\text { length, width } \\
\text { Unit: }[\mathrm{cm}]\end{array}$ & $\begin{array}{l}\text { How much } \\
\text { is the } \\
\text { length of } \\
\text { the side? } \\
\text { [Pattern 3] }\end{array}$ & Unknown & 2 \\
\hline 2 & $\begin{array}{l}\text { Oxygen production } \\
\text { equation with } \\
\text { hydrogen peroxide }\end{array}$ & $\begin{array}{l}\text { Oxygen } \\
\text { productio } \\
\mathrm{n} \\
\text { equation }\end{array}$ & $\begin{array}{l}\text { How to write the } \\
\text { equation for oxygen } \\
\text { production from } \\
\text { hydrogen peroxide }\end{array}$ & $\begin{array}{l}\text { Entity: hydrogen } \\
\text { peroxide } \\
\text { Action: oxygen } \\
\text { production } \\
\text { Entity: oxygen }\end{array}$ & $\begin{array}{l}\text { equation } \\
\text { for oxygen } \\
\text { production }\end{array}$ & Yes & 2 \\
\hline 3 & $\begin{array}{l}\text { When a car } \\
\text { suddenly starts, } \\
\text { passengers will } \\
\text { move backward, } \\
\text { because they are } \\
\text { under the force of } \\
\text { inertia }\end{array}$ & NULL & $\begin{array}{l}\text { "When a car suddenly } \\
\text { starts, passengers will } \\
\text { move backward, because } \\
\text { they are under the force } \\
\text { of inertia". Why is the } \\
\text { sentence wrong? }\end{array}$ & $\begin{array}{l}\text { Entity: inertia } \\
\text { Action: move } \\
\text { backward }\end{array}$ & $\begin{array}{l}\text { Why is the } \\
\text { sentence } \\
\text { wrong? }\end{array}$ & Unknown & 2 \\
\hline
\end{tabular}

\section{RESULTS AND DISCUSSION}

We selected $80 \%$ of the 41,532 data as a training dataset. The remaining $20 \%$ were used as test data. Two baselines were selected: LR (Logistic Regression) with pointwise strategy and LR + DNN with point-pairwise strategy. Neither baseline considers knowledge features. The experimental results are shown in Figure 4:
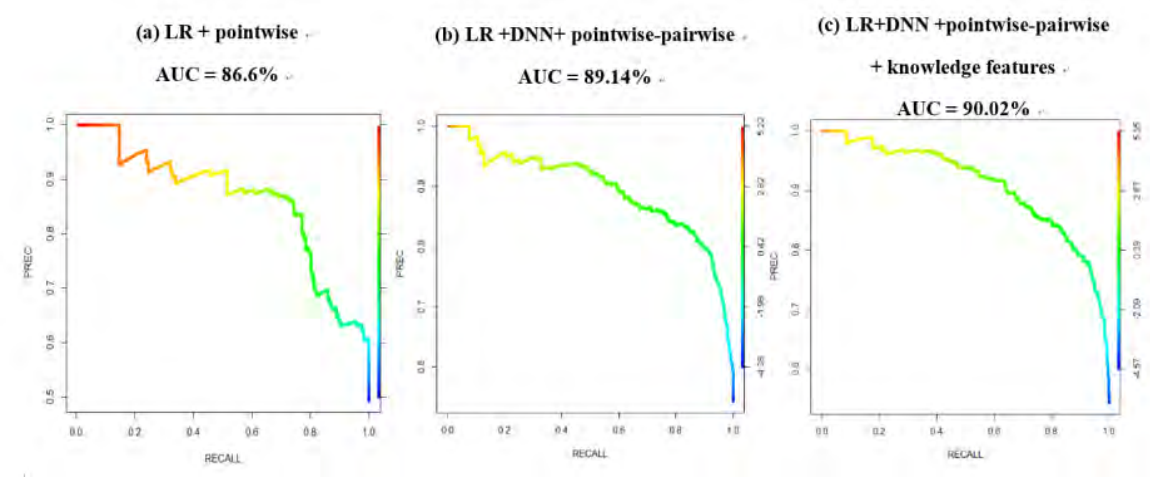

Figure 4. Experimental results of the proposed model 
In the graphs shown in Figure 4, the abscissa represents Recall, and the ordinate represents Precision. The Area Under Curve (AUC) of LR + pointwise strategy is $86.6 \%$. This is improved upon by both LR + DNN (AUC $=89.14 \%)$, and $\mathrm{LR}+\mathrm{DNN}$ with knowledge features $(\mathrm{AUC}=90.02 \%)$. Another important benefit of the proposed model is that it fluctuates less than the other models, making it a more useful predictor.

When we set a threshold of $30 \%$ on recall, the precision of the three models was $92 \%, 94 \%$ and $97 \%$ respectively. We also selected several cases to further illustrate the performance of the proposed model, as shown in Table 3.

Table 3. Case Studies of the proposed model with recall at $30 \%$

\begin{tabular}{|c|c|c|c|}
\hline Query & LR & $\mathrm{LR}+\mathrm{DNN}$ & LR + DNN + Knowledge Features \\
\hline $\begin{array}{l}\text { How many usages does } \\
\text { "Ji" have? }\end{array}$ & $\begin{array}{l}\text { How many usages does } \\
\text { "De" have? }\end{array}$ & $\begin{array}{l}\text { How many usages } \\
\text { does "De" have? }\end{array}$ & $\begin{array}{l}\text { What is the specific usage of "Ji } \\
\text { " in Chinese? }\end{array}$ \\
\hline $\begin{array}{l}\text { Why should diazo } \\
\text { reaction be carried out } \\
\text { at low temperatures? }\end{array}$ & $\begin{array}{l}\text { Why should diazo } \\
\text { reaction be carried out } \\
\text { at low temperatures? }\end{array}$ & $\begin{array}{l}\text { Why does diazo } \\
\text { reaction not need } \\
\text { to be carried out } \\
\text { under strong acid } \\
\text { conditions? }\end{array}$ & $\begin{array}{l}\text { What is diazo reaction? Why } \\
\text { should it be carried out at low } \\
\text { temperatures? }\end{array}$ \\
\hline $\begin{array}{l}\text { In a circle with a } \\
\text { diameter of } 4 \mathrm{~cm} \text {, draw } \\
\text { the largest square. }\end{array}$ & $\begin{array}{l}\text { Draw the largest circle } \\
\text { in a square with a } \\
\text { length of } 4 \mathrm{~cm} .\end{array}$ & $\begin{array}{l}\text { Draw the largest } \\
\text { square in a circle. }\end{array}$ & $\begin{array}{l}\text { Here is a circle with a diameter of } \\
4 \mathrm{~cm} \text {. Please draw the largest } \\
\text { square in the circle and calculate } \\
\text { its area. }\end{array}$ \\
\hline
\end{tabular}

As can be seen in Table 3, the proposed model performs better on cases where query and title have the same meaning but are expressed in different ways. By taking into consideration entities related to knowledge points , the model can identify important differences and make correct judgement, while other state-of-art algorithms still depend on term matching similarity. Currently, knowledge features can only contribute a $1 \%$ improvement, because the construction of the knowledge graph is still at an early stage. With continuous construction of the knowledge graph, the model will be improved significantly.

For parameter assignment, we set learning rate at 0.02 , batch at 128 , margin of hinge-loss at 0.1 , and sampling-rate of shuffle at 0.1 . The training curve can be seen below in Figure 5.

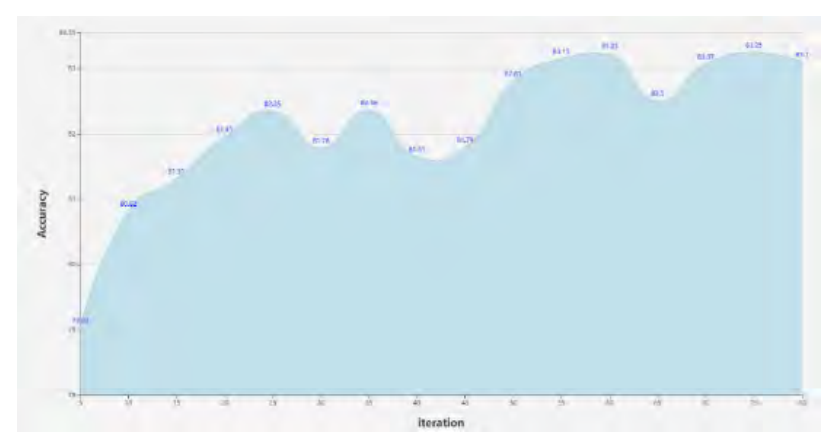

Figure 5. Accuracy statistics of training process

\section{CONCLUSION}

In this research, we used a two-stage cascading model with knowledge features to optimize a test-question/answer search task. In Stage 1, we used a log of 41,532 clicked records and 207,660 unclicked records to generate a 200,000 pairwise dataset. This was used to train a DNN model to recognize semantic correlation through users' preference patterns, thus further improving generalizability. In stage 2 , we designed and incorporated knowledge features to improve semantic understanding by the model, of different questions. The combination of Stages 1 and 2 could not only accurately interpret semantic information relating to the 
questions, but also improved generalizability using the large-scale user log. One challenge in information retrieval is generalizing from a precise semantic model. The proposed model helps to address that challenge.

In future, we would like to design more complete and accurate knowledge features, and create a domain knowledge graph for test-questions/answers. In addition, we are seeking to incorporate LR and DNN into a unified framework to realize collaborative learning, which can achieve a dynamic balance between generalizability and accurate semantic understanding.

\section{ACKNOWLEDGEMENT}

This work was supported by Chinese National Youth Foundation Research (Grant No: 61702564), Talent Scientific Research Foundation of Sun Yat-sen University (20000-18831102).

\section{REFERENCES}

Danchen Zhang, Daqing He, 2018. Can Word Embedding Help Term Mismatch Problem? - A Result Analysis on Clinical Retrieval Tasks. International Conference on Information. Sheffield, UK, pp 402-408.

Gómez-Adorno, H., Pinto, D. and Vilarino, D., , 2013. A Question Answering System for Reading Comprehension Tests. Mexican Conference on Pattern Recognition, Spring, Berlin, Heidelberg, pp 354-363.

Hinton G. E, Salakhutdinov R. R, 2006. Reducing the dimensionality of data with neural networks. Science, Vol. 313, No. 5786, pp 504-507.

P.-S.Huang, X.He, J.Gao, et al, 2013. Learning deep structured semantic models for web search using clickthrough data. Proceedings of the 22nd ACM international conference on Conference on Information and Knowledge Management. San Francisco, USA, pp 2333-2338.

Jiafeng Guo, Yixing Fan, Qingyao Ai, W. Bruce Croft, 2016. A deep relevance matching model for Ad-hoc retrieval. proceedings of the 25th ACM International conference on Conference on Information and Knowledge Management. Indianapolis, USA, pp 55-64.

Jun Yu, Dacheng Tao, Meng Wang, Yong Rui, 2015. Learning to Rank Using User Clicks and Visual Features for Image Retrieval. IEEE Transactions on Cybernetics, Vol. 45, No. 4, pp 767-779.

G.Salton, A.Wong, and C.-S.Yang, 1975. A vector space model for automatic indexing. Communications of the ACM, Vol. 18, No. 11, pp 613-620.

Sunil Mohan, Nicolas Fiorini, Sun Kim, Zhiyong Lu, 2017. Deep Learning for Biomedical Information Retrieval: Learning Textual Relevance from Click Logs. Proceeding of the 16th Biomedical natural language processing. Vancouver, Canada, pp 222-231.

Timur Osadchiy, Ivan Poliakov, Patrick Olivier, et al, 2019. Recommender system based on pairwise association rules. Expert Systems with Applications, Vol. 115, pp 535-542.

Xiao Bai, Erik Ordentlich, Yuanyuan Zhang,et al, 2018. Scalable Query N-Gram Embedding for Improving Matching and Relevance in Sponsored Search. Proceedings of the 24th ACM SIGKDD International Conference on Knowledge Discovery and Data Mining. London, UK, pp 52-61.

W. Zhang, R. Jones, 2007. Comparing click logs and editorial labels for training query rewriting. 16th International World Wide Web Conferences. Banff, Canada, pp 2-5. 